\title{
Laser-Enhanced Short-Circuiting Metal Transfer in GMAW
}

\section{Laser irradiation enhanced short-circuiting metal transfer under CC power mode to control transfer frequency and improve process stability}

\author{
BY S. J. CHEN, Y. Z. JIA, J. XIAO, AND T. WEN
}

\begin{abstract}
Previously, pulsed laser-enhanced gas metal arc welding (GMAW) was studied toward the goal of realizing current-independent free-flight metal transfer. However, controlled low-spatter or even spatter-free short-circuiting transfer would be more preferred in applications requiring even lower heat input. Thereby, laser-enhanced metal transfer was expanded to short-circuiting transfer by using a relatively short arc. A constant current (CC) power source was employed to avoid the current boost during the short-circuiting period. The arc variables and metal transfer dynamics were synchronously recorded. The short-circuiting transfer behavior under constant and pulsed laser irradiation were both observed and analyzed in this study. The experimental results show the laser irradiation does enhance the short-circuiting metal transfer under CC power mode in the ways of controlling the transfer frequency and improving the process stability. The role of the laser recoil force changes to first actively initiate and then terminate the short-circuiting process between the droplet and molten pool. The start and end of the short-circuiting process both become controllable. A sufficiently high short-circuiting current is no longer needed to severely pinch the droplet and thus guarantee termination of the short circuit. On the other hand, welding spatter produced by the current boost in constant voltage (CV) power mode is nearly eliminated. What is more, the transfer frequency can be precisely controlled when using pulsed laser irradiation. A kind of one-droplet-per-pulse (ODPP) short-circuiting transfer, almost without spatter, is obtained as desirable.
\end{abstract}

\section{KEYWORDS}

- Laser Irradiation • Short-Circuiting Transfer

- Current Waveform • One-Droplet-Per-Pulse Transfer

\section{Introduction}

Gas metal arc welding (GMAW) is currently the most widely used arc welding method in manufacturing, which is attributed to its high productivity by using a consumable wire electrode and its good compatibility to automatic/robotic welding.

Short-circuiting GMAW (GMAW-S) is a variant of GMAW where the wire-droplet compound periodically shorts the circuit with the weld pool (Refs. 1-3). As a special variant of GMAW, GMAW-S distinguishes itself by metal transfer from typical free-flight transfer GMAW. Because of the shortcircuiting metal transfer, the arc in GMAW-S is periodically terminated and then reignited. The typical advantage of GMAW-S, compared with free-flight-transfer GMAW, is the lower heat input to the workpiece. The obstacle of conventional GMAW-S for stable arc and consistent metal transfer is the lack of controllability on its short-circuiting metal transfer. If appropriately controlled to significantly reduce or even eliminate spatter to obtain smooth, gas tungsten arc welding (GTAW) like surface tension absorption transfer, GMAW-S promises clean operation with minimal fume/ smoke as with GTAW, but at GMAW speeds.

Conventional GMAW-S generally uses a constant voltage (CV) power source associated with constant wire feed speed. The adoption of a CV power source helps to maintain the stability of the arc length no matter whether in shortcircuiting or free-flight-transfer mode. Setting a relatively small arc voltage can keep the arc length short and thus ensure the droplet would always short circuit with the weld pool when it grows to a relatively small size. On the other hand, particularly for short-circuiting transfer, the $\mathrm{CV}$ power source plays a crucial role in guaranteeing the break of the shortcircuiting liquid bridge and reignition of the arc, since the welding current of a $\mathrm{CV}$ power source would rapidly boost once short-circuiting occurs. Such a current boost affects in two ways: 1) to produce a strong pinch effect on the shortcircuiting bridge to forcedly neck it; 2) to induce a local explosion of the short-circuiting bridge due to the high current density going through the necked bridge. With the assistance 


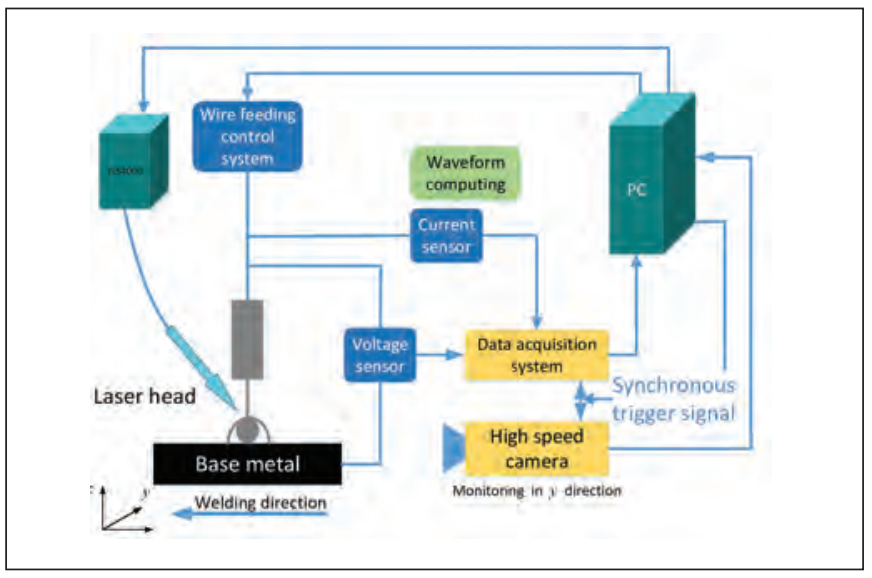

Fig. 1-Sketch of laser-enhanced short-circuiting metal transfer system.

of these two effects of current boost and the surface tensiondriven absorption from the weld pool, the end of short-circuiting, i.e., the reigniting of the arc, is solidly ensured. However, in the view of fine control of short-circuiting transfer, the pinch effect and surface tension absorption are both appreciated but the explosion effect will not be due to the spatter generated (Refs. 4, 5). However, using a constant current (CC) power source with a variable-speed wire feed system can provide a constant heat input to ensure stable and quantitative melting of the wire, and the real waveform of the welding current can be optimized for each specific application. However, the metal transfer independent of the current in CC GMAW is a challenge. Especially for short-circuiting transfer, it is going to be unstable due to the absence of high shortcircuiting current if no external force is introduced.

Based on this analysis, one can conclude that fine control of GMAW-S lies in the way of ensuring robust initiation and termination of the drop-pool short-circuiting process. Conventional GMAW-S adopting a CV power source is clearly an effective way but also produces an unstable arc and inconsistent metal transfer, and it always has relatively severe spatter and poor bead formation. During the past decades, the two major successes in modification of GMAW-S have been surface tension transfer (STT) (Ref. 6) and cold metal transfer (CMT) (Refs. 7-10). The STT method aims at precise control of current waveform (Refs. 11-14). Its core purpose is to rapidly reduce the high short-circuiting current to a base level at the very moment the high short-circuiting current has sufficiently pinched the liquid bridge, thus it can be certainly absorbed and detached to the weld pool by surface tension, meanwhile the high-current-induced explosion is avoided. In this way, the short-circuiting transfer becomes quite smooth and has very low spatter.

Instead of current waveform control, CMT seeks a mechanical enhancement of the metal transfer. When the droplet contacts the molten pool, the wire is instantly pulled away from the pool, and thus the droplet is detached and the arc is reignited. Then the wire is fed toward the pool again until the next short-circuit occurs. Cold metal transfer can achieve a stable short-circuiting transfer under a small current with almost no spatter. The CMT method requires a complicated wire feed system and the motors limit the dynamic performance.

Additionally, Zhang et al. proposed a double-electrode

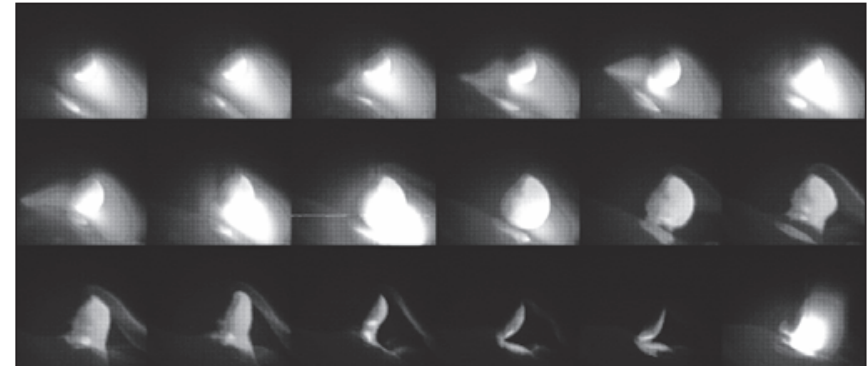

Fig. 2 - Short-circuiting metal transfer behavior under the condition of $80 \mathrm{~A}$.

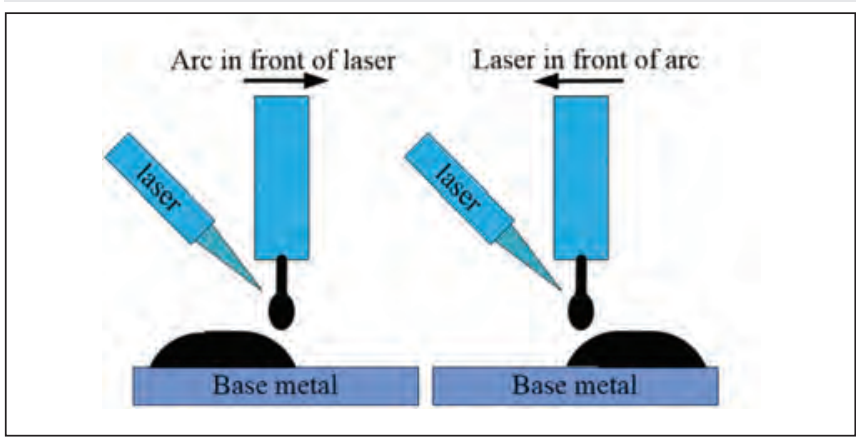

Fig. 3 - Illustration of welding direction.

GMAW process and control system, attempting to improve controllability while maintaining the low heat input of GMAW-S (Ref. 15). A bypass arc was established between the wire and an additional electrode. Thereby, the total current flowing through the wire and the base metal could be separately controlled. The metal transfer can also be enhanced by the bypass arc. However, the metal transfer was only of freeflight mode.

Researchers at the University of Kentucky have proposed a laser-enhanced GMAW process (Ref. 16). A CC power source was adopted, and a laser beam was aimed at the droplet to partially vaporize it, thus generating a recoil force to detach the droplet (Refs. 17-19). Drop spray transfer was achieved in the current range of 40-120 A with 0.8-mm ER70s-6 wire and pure argon shielding gas.

In this paper, the authors are trying to expand the laser irradiating method to short-circuiting metal transfer control. By properly shortening the arc, the pendant droplet may be forcedly elongated/driven to short circuit with the weld pool. And the laser detaching effect still works after it, i.e., the short-circuiting bridge would be forcedly cut off by the laser. Thereby, both the initialization and termination of the shortcircuiting process can be actively controlled. This paper is going to experimentally verify the proposed laser-driven shortcircuiting metal transfer. Also, the effect of the laser parameters will be analyzed.

\section{Experimental Setup}

The experimental system was composed of a laser system, welding system, high-speed camera, data acquisition, and control unit, as shown in Fig. 1. Available functions are 1) laser spot size, incident position, and incident angle can be finely adjusted; 2) arc length in constant current (CC) 

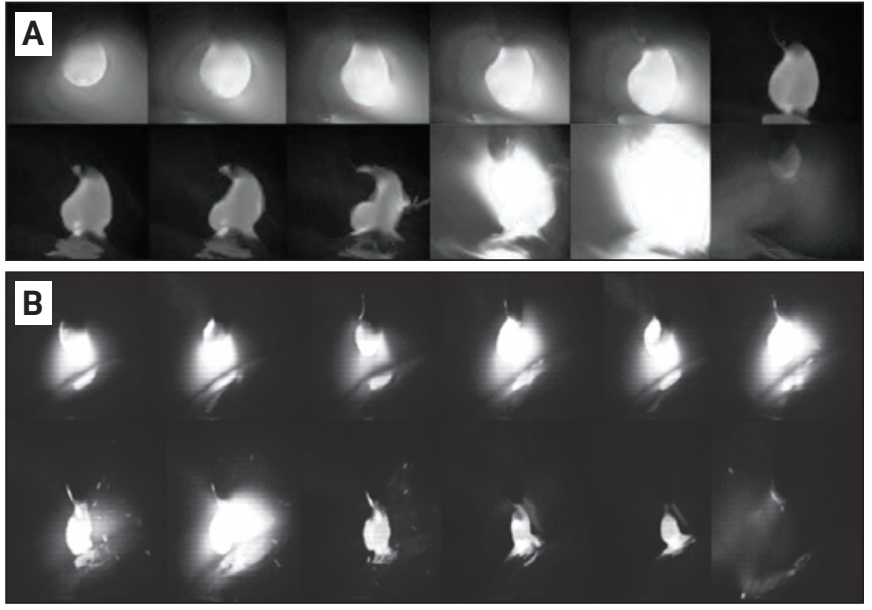

Fig. 4 - Effect of welding direction of laser-enhanced shortcircuiting transfer: A - Laser behind; B - laser ahead.

mode can be controlled; and 3) synchronous acquisition of droplet transfer image and welding electrical signal. A CC power source was adopted. An IPG YLS4000 fiber laser was used in the experiments for laser pulse output. The maximum power of the laser was $4000 \mathrm{~W}$ in continuous wave (CW) mode, and it could be modulated into pulsed mode. The laser spot diameter was about $0.5 \mathrm{~mm}$. The welding wire was ER70S- 6 mild-steel wire with a diameter of 0.8 $\mathrm{mm}$. Bead-on-plate welding was conducted on mild-steel plates. The contact tip-to-workpiece distance (CTWD) was set at $12 \mathrm{~mm}$. Pure argon was used as the shielding gas at a flow rate of $15 \mathrm{~L} / \mathrm{min}$. The high-speed camera worked at a frame rate of 3000 frames per second (fps) in axis. The laser incident point on the droplet was controlled by matching the arc length. Test parameters are shown in Table 1.

\section{Results and Discussion}

\section{Short-Circuiting Transfer of Low-Current GMAW}

Experiment 1 first analyzed the short-circuiting transfer behavior in conventional GMAW. The current was $80 \mathrm{~A}$. The frequency of droplet transfer increased with the increase in wire feeding speed. Figure 2 shows typical short-circuiting
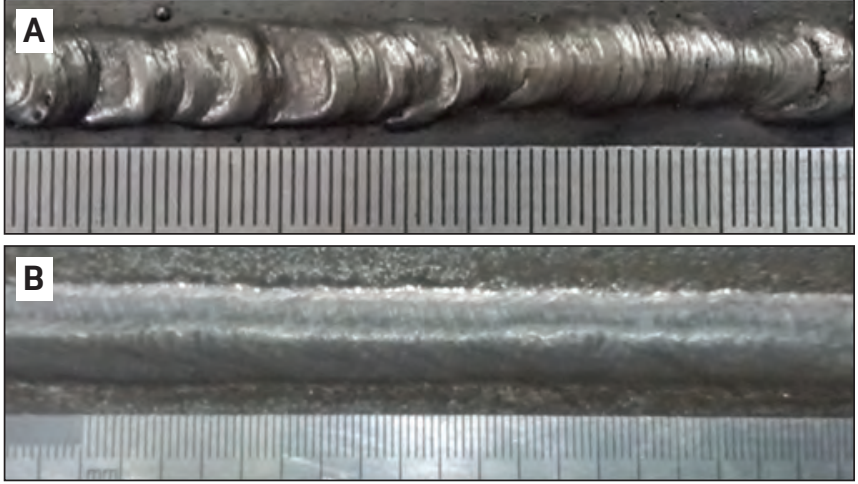

Fig. 5 - Comparison of bead formation in Experiments 1 and 3; A - Bead formation of GMAW-S with CC power source; $B-$ bead formation of laser-enhanced GMAW-S with CC power source.

transfer behavior when wire feed speed is $2.0 \mathrm{~m} / \mathrm{min}$. The transfer frequency was about $4 \mathrm{~Hz}$. The droplet diameter at the detaching moment was $2.1 \mathrm{~mm}$. With the increase in wire feed speed, the welding process became unstable. The wire tended to dip into the workpiece/weld pool solidly and then terminate the welding process due to the absence of current boost in CC mode. Stable short-circuiting metal transfer in CC-GMAW needs external control.

\section{Effect of Welding Direction}

The CW laser-enhanced short-circuit transfer was first verified in two weld directions. Since the laser installation was fixed, the laser-droplet-pool profiles changed with the welding direction - laser behind or laser ahead — as illustrated in Fig. 3. The typical metal transfers in Experiments 2 and 3 are shown in Fig. 4A and B, respectively. First, one can see the droplet is forced to contact the weld pool by the laser recoil force. The short-circuit is initialized. After such a forced shortcircuiting, the laser still plays the role of forcedly necking and further cutting off the short-circuit bridge to reignite the arc, and without the high short-circuiting current-induced explosion that is always associated with traditional GMAW-S. This experimental result shows the laser irradiation method does have the ability to force both the start and end of the shortcircuiting transfer. The stability and robustness of shortcircuit metal transfer thus can be guaranteed. Moreover, such

Table 1- Arc and Laser Parameters

\begin{tabular}{|c|c|c|c|c|c|c|}
\hline No. & $\begin{array}{l}\text { Current } \\
(\mathrm{I} / \mathrm{A})\end{array}$ & $\begin{array}{c}\text { Base } \\
\text { Current } \\
\left(I_{h} / A\right)\end{array}$ & $\begin{array}{l}\text { Laser Peak } \\
\text { Power } \\
(P / W)\end{array}$ & $\begin{array}{c}\text { Pulse } \\
\text { Frequency } \\
(f / H z)\end{array}$ & $\begin{array}{l}\text { Pulse } \\
\text { Width } \\
\text { (t/ms) }\end{array}$ & $\begin{array}{l}\text { Laser-Arc } \\
\text { Positioning }\end{array}$ \\
\hline 1 & 80 & - & 0 & - & - & - \\
\hline 2 & 80 & - & 2000 & - & - & Laser behind \\
\hline 3 & 80 & - & 2000 & - & - & Laser ahead \\
\hline 4 & 80 & - & 2000 & - & - & Laser ahead \\
\hline 5 & 80 & - & 2000 & 25 & 2 & Laser ahead \\
\hline 6 & 80 & - & 2000 & 25 & 6 & Laser ahead \\
\hline 7 & 80 & - & 2000 & 25 & 10 & Laser ahead \\
\hline 8 & 80 & - & 2000 & 50 & 6 & Laser ahead \\
\hline 9 & 80 & - & 2000 & 75 & 6 & Laser ahead \\
\hline 10 & 80 & 30 & 2000 & 75 & 6 & Laser ahead \\
\hline
\end{tabular}



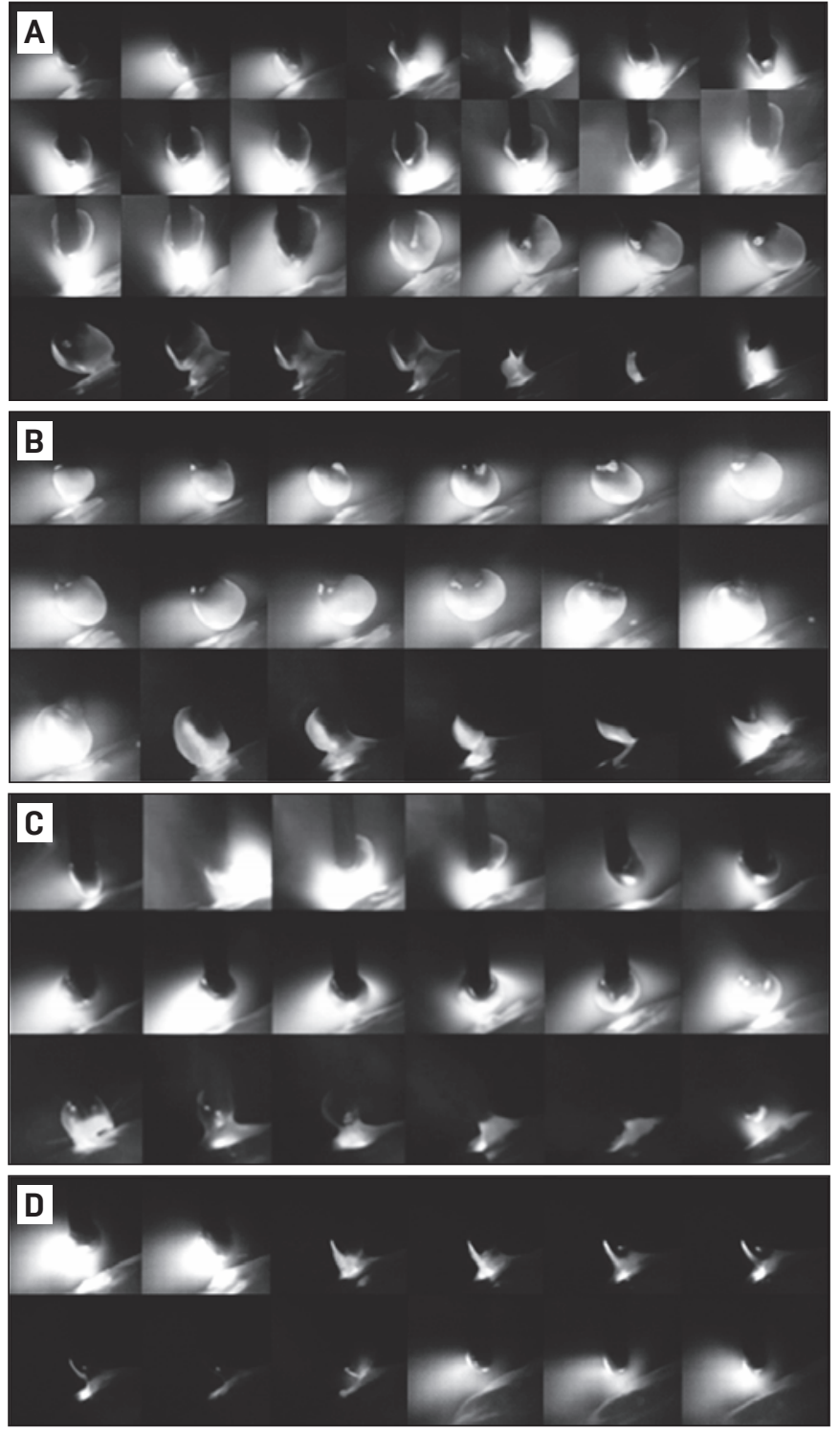

Fig. 6 - Illustration of laser incident point on droplet: A Metal transfer with laser aimed at drop mid-bottom; B metal transfer with laser aimed at drop middle; $\mathrm{C}-$ metal transfer with laser aimed at drop mid-top; D - metal transfer with laser aimed at drop neck.

controllability is exactly the core of short-circuit metal transfer control. Figure 5 shows the bead formation of Experiments 1 and 3 , respectively. It can be seen that the bead formation of Experiment 3, in which the laser is irradiating the droplet to actively control the short-circuiting transfer, is much smoother and more uniform. It is indicated that the laserdriven method significantly increases the short-circuiting transfer frequency and the process is robust.

However, one can notice the difference between Fig. 4A and B. In the case of laser behind, the droplet is pushed away from the front surface of the weld pool. Although the droplet is elongated by the laser recoil force, it cannot contact the weld pool/workpiece until it grows large enough. In the case of laser ahead, the droplet is pushed toward the front surface of the weld pool. It looks like the droplet is heading into the weld pool. The distance between the wire tip and short-circuiting
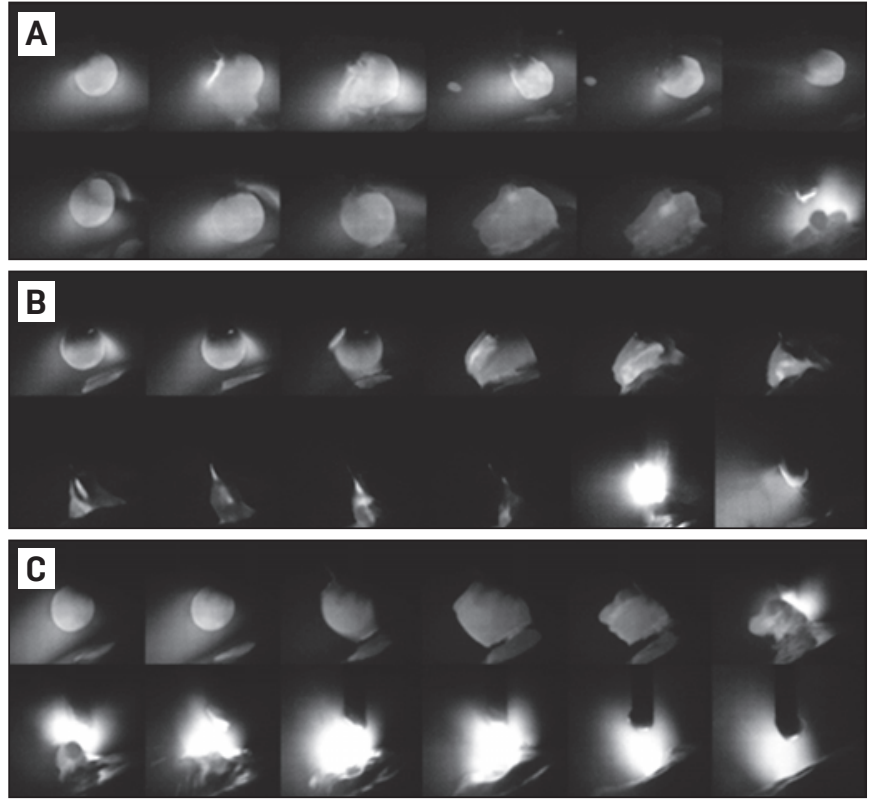

Fig. 7-Effect of different pulse widths under the condition of $25 \mathrm{~Hz}$ on metal transfer: $A-2-m s$ pulse laser width; $B-$ 6-ms pulse laser width; $C-10$-ms pulse laser width.

point on the pool surface is shorter. As such, the droplet is forced to short circuit with the weld pool at a relatively smaller size. The droplet diameter measured at the very short-circuiting start moment of Experiment 2 is about $1.6 \mathrm{~mm}$, but approximately $1.2 \mathrm{~mm}$ in Experiment 4 with laser ahead of the arc in the welding direction.

\section{Effect of Laser Incident Position}

Under the irradiation of the high-energy-density laser spot, a vapor jet is produced against the droplet. The direction of the vapor is not the same as the laser incident direction, but actually in the normal direction of the local droplet surface that is irradiated by the laser spot (Refs. 20, 21). In this case, the most important laser incident parameter would be the laser incident position on the droplet. Small variations in the laser incident point would not affect the metal transfer behavior significantly.

In Experiment 4, the laser installation is fixed, and the irradiated position of the droplet is changed by controlling the arc length. As illustrated in Fig. 6A-D, the droplet incident point is controlled moving from the droplet mid-bottom to the neck. As shown in Fig. 5A, the arc is relatively longer than that of the normal GMAW-S process.

Without the laser irradiation, the droplet would grow large to contact the weld pool. Because the droplet mass center is higher than the laser incident position (mid-bottom), the main effect of the laser recoil force is to swing the droplet. Such a swinging effect is more intensive when the droplet is still relatively small, but not intensive enough to detach the droplet in the repelled mode like that in $\mathrm{CO}_{2}$ welding. Consequently, the droplet keeps growing, and with the increase in the droplet size, the nonaxisymmetric mass transfer is restricted and the wire-axial elongating effect gets stronger. The recoil force changes to a detaching force, 

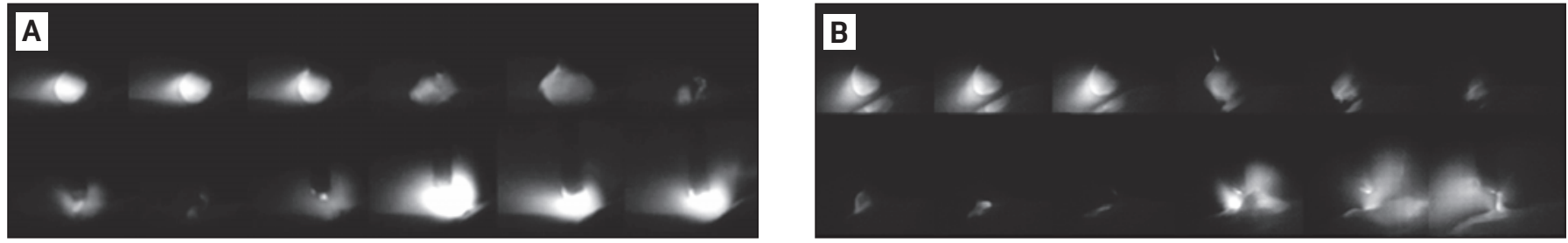

Fig. 8- Effect of different pulse frequencies on metal transfer: A - 50-Hz pulse frequency; $B-75-\mathrm{Hz}$ pulse frequency.

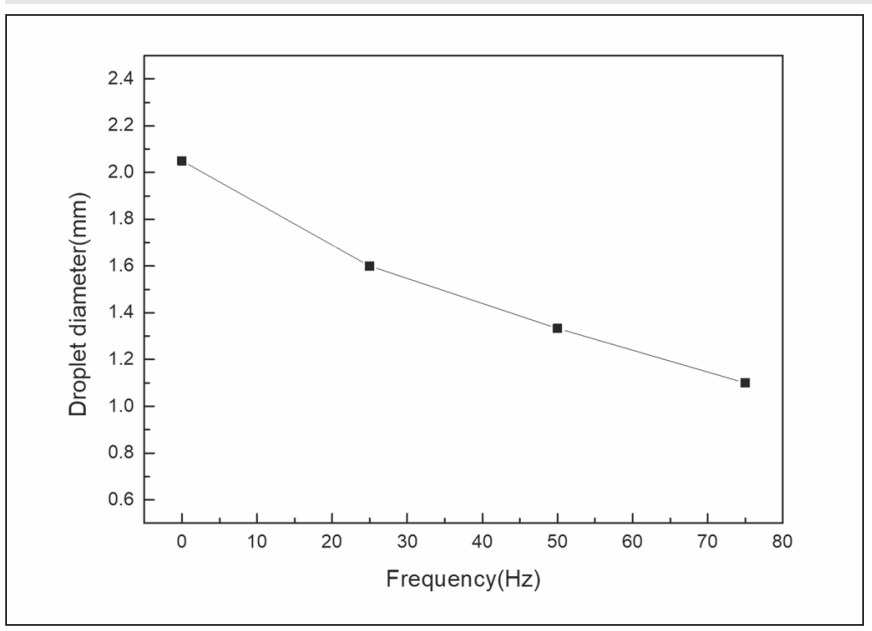

Fig. 9-Droplet diameters of different frequencies.

which elongates the droplet and thus initiates its shortcircuiting with the weld pool.

When the laser spot irradiates the middle of the droplet, the height of the droplet mass center is approximately equal to the laser incident position. The droplet is subjected to the recoil force of unpredictable direction and thus results in nonaxisymmetric mass transfer. The transfer behavior is very similar with the case, as Fig. 6B shows. The droplet is first repelled and then forced short-circuited with the pool and then forced detached. The detached droplet is still a relatively large size.

When the laser irradiates the mid-top of the droplet, as shown in Fig. 6C, the recoil force mainly promotes the droplet transfer, which produces axial droplet elongation, and still radial drift due to the use of a single-side laser. But they are not strong enough to make the droplet contact with the molten pool when the droplet is too small. When the droplet grows, it is forced to contact with the molten pool and the force detaches it. However, the detached droplet size is apparently smaller.

When the laser beam irradiates the neck of the droplet, as shown in Fig. 6D, the droplet elongation is strong enough to force the droplet to contact with the molten pool under a smaller size, of $1 \mathrm{~mm}$. The average transfer frequency is about $80 \mathrm{~Hz}$. This result again proves that the CW laser irradiation method can generate adequate laser recoil force to replace the electromagnetic force to neck and detach the droplet in shortcircuit mode. What is more, the laser also can actively initiate the short-circuit with a relatively long arc and small droplet.

\section{Effect of Laser Peak Pulse Width}

Experiments 5-7 are conducted with pulsed laser irradiation. The laser pulse frequency was fixed at $25 \mathrm{~Hz}$, but the laser peak width is 2,6 , and $10 \mathrm{~ms}$, respectively. The laser was aimed at the droplet neck position. Figure 7 shows the droplet transfer behavior under pulsed laser irradiation in those experiments. As shown in Fig. 7A, the droplet deforms obviously due to the laser recoil force, and the droplet is stretched and shifted. However, due to the lack of laser peak width, the duration of recoil force is not long enough to push the droplet to contact the pool. Two laser pulses are needed to facilitate the desired short-circuiting transfer. In Fig. 7B, with the increase in laser pulse width, the duration of laser recoil force is long enough to first force the droplet short-circuiting and then cut off the short-circuiting liquid bridge. Desired active shortcircuiting transfer is obtained. When the laser pulse width is further increased to $10 \mathrm{~ms}$, as shown in Fig. 7C, the laser still presents after the droplet short-circuit process is completed. At the very moment, the laser is actually aimed at the weld pool since the next droplet has just been initiated. The vaporization of the weld pool would help to enhance the arc stability after the arc re-ignition. These results show the pulsed laser irradiation can indeed lead to the desired control of active shortcircuiting start and end, but a minimum laser peak duration is required to obtain one-pulse-per-drop short-circuiting transfer. A little excessive pulse width contributes to shortening the short-circuiting duration; it is $3.6 \mathrm{~ms}$ and $3.0 \mathrm{~ms}$ in Experiments 6 and 7 .

\section{Effect of Laser Pulse Frequency}

The results of Experiments 6, 8, and 9 are referred to analyze the effect of laser pulse frequency. The laser peak pulse in these three experiments is set at $6 \mathrm{~ms}$. As can be seen from Fig. 8, with the increase in the laser pulse frequency, the metal transfer mode is the desired active short-circuiting transfer, but the critical droplet size decreases, as shown in Fig. 9. These results further prove that the pulsed laser irradiation method can produce enough recoil force to force the droplet to be short-circuited and then detached, and the verified $75-\mathrm{Hz}$ onepulse-per-droplet transfer demonstrates satisfactory dynamic performance.

\section{Analysis of Arc Variables}

The recorded arc variable waveforms of Experiments 1, 4, 6, 8, and 9 are shown in Fig. 10A-E. Figure 10A is recorded from Experiment 1, without laser irradiation. The irregular arc voltage drop shows the uneven period between each shortcircuiting transfer. And there are some random instantaneous short circuits between each transfer cycle. The current waveform shows an unexpected sharp boost during the short -circuiting period even when the power supply works in CC mode. The maximum short-circuiting current is near $125 \mathrm{~A}$, much lower than that of traditional CV GMAW-S. However, 

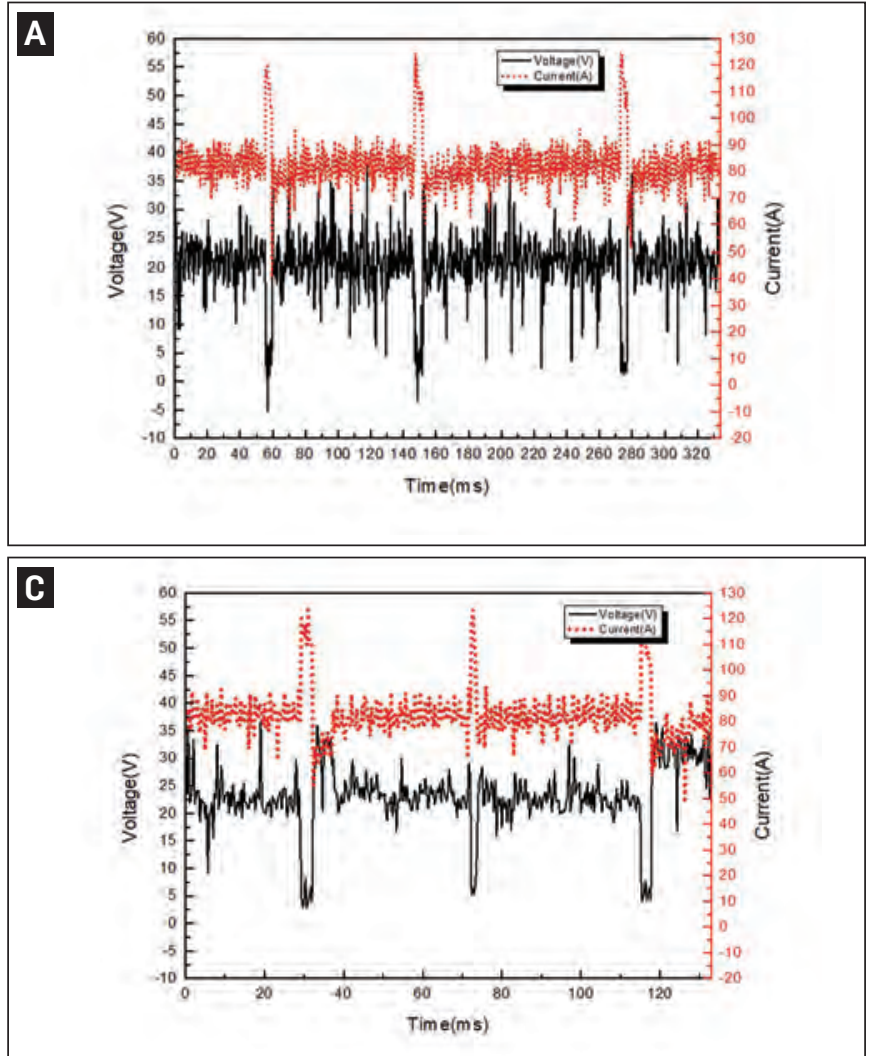

Fig. $10-$ Analysis of current and voltage signal acquisition: $A-80$ A without laser; $B-80 \mathrm{~A}$ with $\mathrm{CW}$ laser; $\mathrm{C}-80 \mathrm{~A}$ with pulse laser of $25 \mathrm{~Hz}$; D $-80 \mathrm{~A}$ with pulse laser of $50 \mathrm{~Hz}$; $\mathrm{E}-80 \mathrm{~A}$ with pulse laser of $75 \mathrm{~Hz}$.

such a relatively weak current boost still produces slight spatter at the very moment of arc reignition. The reason for such a current increase should lie in the power source itself.

Figure 10B corresponds to the results of Experiment 4, where the laser works in CW mode and aims the droplet neck position. Each negative pulse of arc voltage represents an occurrence of laser-driven short-circuiting transfer. However, it can be clearly seen that the time interval between each transfer is severely uneven, and the width of the negative pulses, measuring the short-circuiting period, is also uneven. As such, the CW laser-driven short-circuiting transfer is guaranteed but not precisely predictable.

Figure 10C corresponds to Experiment 6, 8, and 9. The only difference is the laser pulse frequency. It can be seen that the metal transfer frequency exactly equals the laser pulse frequency. Even the pulse of the negative pulse of arc voltage in each experiment is approximately even.

As mentioned in the laser subsection, the current still jumps during the short-circuiting period and generates spatter. However, it is possible to reduce the short-circuit current if it is intentionally reduced to a base level $(30 \mathrm{~A})$ right after the laser pulse is emitted. In this way, the heat input to the weld pool can also be reduced. As shown in Fig. 11, the current and laser waveforms are synchronized. Figure 12 shows the shortcircuiting transfer with a laser pulse frequency of $75 \mathrm{~Hz}$ and a pulse width of $6 \mathrm{~ms}$. When the laser is modulated, the current is reduced to a base value of $30 \mathrm{~A}$. No spatter is observed during the short-circuiting transfer process. However, as can be
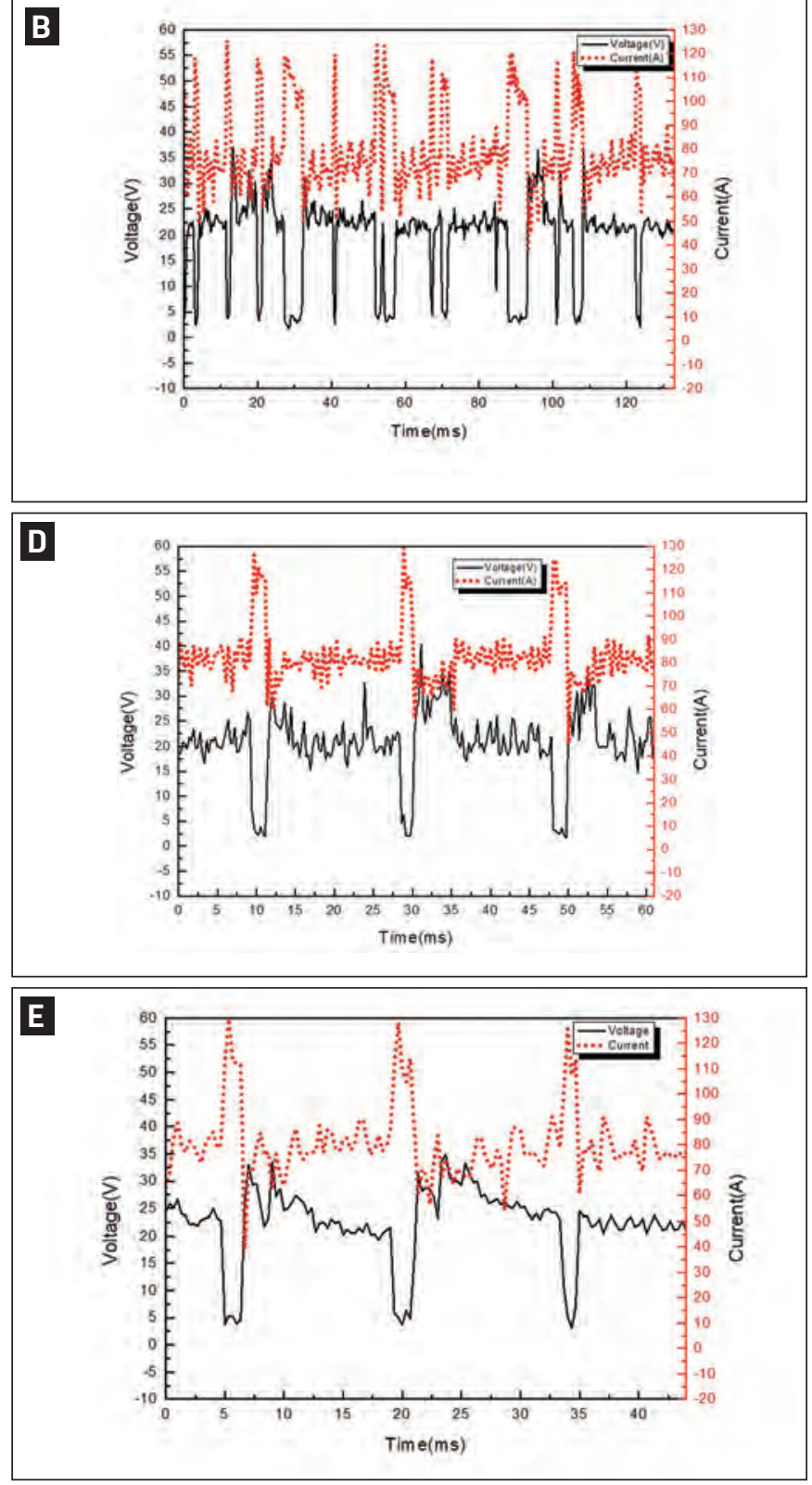

seen from the figure, the current keeps at base for a little while after the arc has been re-ignited. Actually, only the falling edge of current needs to be synchronized with the laser pulse; the rising edge does not. The base current duration could fine adjust upon heat input preference.

\section{Conclusions}

1) Both CW and pulsed laser irradiation can actively control the short-circuiting transfer behavior. The start and end of short-circuiting between the droplet and weld pool both become a laser-driven behavior.

2) Pulsed laser irradiation is optimal since one-drop-perpulse short-circuiting transfer can be achieved in this mode. The transfer frequency and droplet size can be controlled by adjusting the laser pulse frequency. The metal transfer frequency at 80-A current could be up to $75 \mathrm{~Hz}$.

3) The process stability can be further improved by com- 


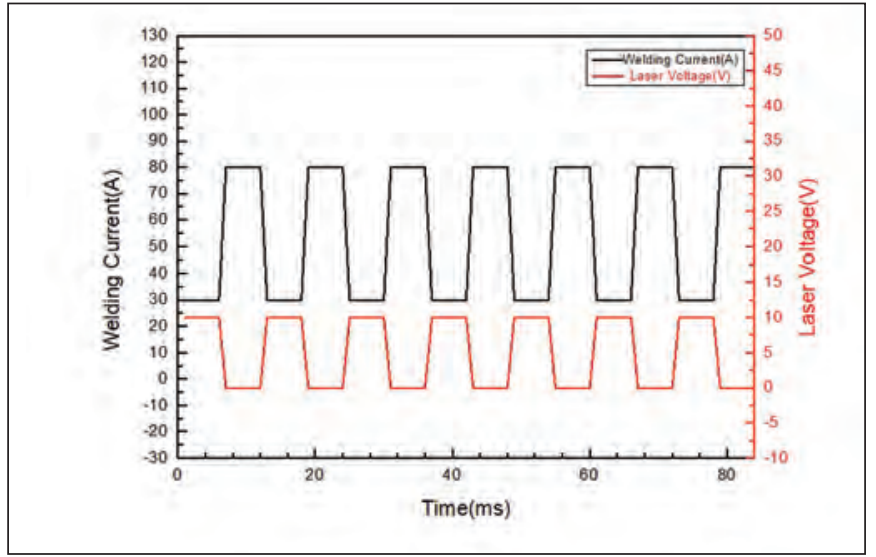

Fig. 11 - Sketch of laser-current cooperative control metal transfer.

bining the pulsed laser irradiation and current waveform control. Spatter during the short-circuiting process can be almost eliminated and the heat input during short-circuiting can be reduced by synchronously reducing the current base when the laser pulse is emitted.

\section{Acknowledgments}

This work was supported by the Natural Science Foundation of China under grants 51505009 and 51575133.

\section{References}

1. Iordachescua, D., and Quintinob, L. 2008. Steps toward new classification of metal transfer in gas metal arc welding. Journal of Materials Processing Technology 202: 391-397. DOI: doi.org/10.1016/j.jmatprotec.2007.08.081

2. Hsu, C., and Stava, E. 1999. Wire extension measurement during short circuiting metal transfer. Metal welding and applications, Proceedings of the Interdisciplinary Symposium. Met. Soc., p. 351-358.

3. Bless, S. J. 1974. Drop transfer in short-circuit welding. J. Phys. D: Appl. Phys. 7D: 526-539. DOI: doi.org/10.1088/0022-3727/7/ $4 / 306$

4. Hermans, M. J. M., and Den Ouden, G. 1999. Process behavior and stability in short circuit gas metal arc welding. Welding Journal 78(4): 137-s to 141-s.

5. Adolfsson, S., Bahrami, A., Bolmsjo, G., and Claesson, I. 1999. On-line quality monitoring in short-circuit gas metal arc welding. Welding Journal 78(2): 59-s to 73-s.

6. Deruntz, B. D. 2003. Assessing the benefits of surface tension transfer welding to industry. Journal of Industrial Technology 19(4): 2-8.

7. Lin, S. B., Fan, C. L., Song, J. L., et al. 2007. Research on CMT welding of nickel-based alloy with stainless steel. China Welding 16(3): 23-26.

8. Zhang, H. T., and Feng, J. C., et al. 2009. The arc characteristics and metal transfer behavior of cold metal transfer and its use in joining aluminum to zinc-coated steel. Materials Science and Engineering 499(2): 111-113. DOI: doi.org/10.1016/j.msea.2007.11.124

9. Wang, J., and Feng, J. C. 2008. Microstructure of Al-Mg dissimilar weld made by cold metal transfer MIG welding. Material Science and Technology 24(7): 827-831. DOI: doi.org/10.1179/ $174328408 \times 278411$

10. Pickin, C. G., and Young, K. 2006. Evaluation of cold metal transfer (CMT) process for welding aluminum alloy. Science and Tech-

\section{$\mathbf{A}$}
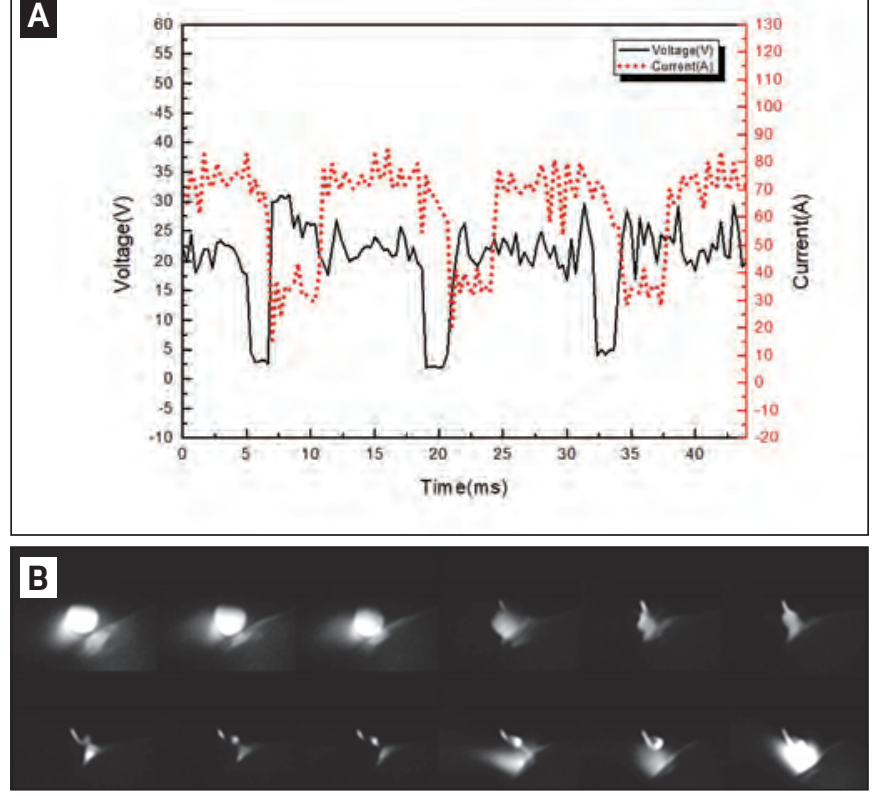

Fig. 12 - Acquisition of short-circuiting transfer signal cooperative driven by laser current, 75- $\mathrm{Hz}$ pulse frequency, 6-ms pulse width, and 30-A base current: A - Acquisition signal of current and voltage; $B$ - high-speed camera pictures of metal transfer.

nology of Welding and Joining 11(5): 583-585. DOI: doi.org/10.1179/ $174329306 \mathrm{X} 120886$

11. Stava, E. K. 1992. System and Method of Short Circuiting Arc Welding. U. S. Patent 5148001.

12. Stava, E. K. 1993. The surface-tension-transfer power source: A new low-spatter arc welding machine. Welding Journal 72(1): 25-29.

13. Kim, Y. S., and Eagar, T. W. 1993. Analysis of metal transfer in gas metal arc welding. Welding Journal 72(6): 269-s to 278-s.

14. Rhee, S., and Kannatey-Asibu, E. 1991. Analysis of arc pressure effect on metal transfer in gas metal arc welding. J. Phys. D: Appl. Phys 24(8): 5068-5075.

15. Li, K. H., Chen, J., and Zhang, Y. M. 2007. Double-electrode GMAW process and control. Welding Journal 86(8): 231-s to 237-s.

16. Huang, Y., and Zhang, Y. M. 2010. Laser-enhanced GMAW. Welding Journal 89(9): 181-188.

17. Huang, Y., and Zhang, Y. M. 2011. Laser-enhanced metal transfer — Part 1: System and observations. Welding Journal 90(10): 183 -s to 190 -s.

18. Huang, Y., and Zhang, Y. M. 2011. Laser-enhanced metal transfer - Part 2: Analysis and influence factors. Welding Journal 90(11): 206-s to 210-s.

19. Shao, Y., and Zhang, Y. M. 2014. Pulsed laser enhanced GMAW. Welding Journal 93(6): 205-s to 214-s.

20. Xiao, J., Chen, S. J., Zhang, G. J., and Zhang, Y. M. 2016. Current-independentmetal transfer by using pulsed laser irradiation - Part 1: System and verification. Welding Journal 95(3): 93-s to 100 -s.

21. Xiao, J., Chen, S. J., Zhang, G. J., and Zhang, Y. M. 2016. Current-independent metal transfer by using pulsed laser irradiation Part 2: Affecting factors. Welding Journal 96(6): 194-s to 201-s.

SHUJUN CHEN, YAZHOU JA, JUN XIAO (jun.xiao@bjut.edu.cn), and TAO WEN are with the Engineering Research Center of Advanced Manufacturing Technology for Automotive Components, Ministry of Education, College of Mechanical Engineering and Applied Electronics, Beijing University of Technology, Beijing, China. 